\title{
Further considerations in deciding between clipping and coiling for cerebral aneurysms
}

\author{
Ralph L Sacco
}

Aneurysmal subarachnoid hemorrhage (SAH) remains a devastating type of stroke that is more often fatal, and more often affects younger persons, than other stroke types. One of the most important approaches to improve outcome after a sentinel SAH is to eliminate the source of the bleeding. The mainstay of therapy has always been the early surgical clipping of an aneurysm. However, potential complications after clipping surgery have led to the exploration of other 'less invasive' techniques. Endovascular procedures have continued to evolve and improve as catheters and coils have become smaller and easier to navigate through the cerebral circulation, and stents have enabled the more-complete obliteration of even wide-necked aneurysms.

When faced with treatment choices, randomized controlled trials are usually the foundation for making evidence-based decisions. The International Subarachnoid Aneurysm Trial (ISAT) was a large trial designed to compare clipping and endovascular treatment among patients with ruptured intracranial aneurysm. The trial showed that coil embolization resulted in a significantly better outcome at 1 year than did clipping, and there was no difference in the 1-year risk of rebleeding from the ruptured aneurysm (Molyneux AJ et al. [2005] Lancet 366: 809-817).

Controversy over the ISAT findings has led to differing patterns of practice. Critics of the trial have noted that the surgical morbidity and mortality were much worse than expected. Even in 'good grade' patients with small anterior circulation aneurysms there was a high risk of poor outcomes or death, and almost $20.8 \%$ of the SAH cases treated surgically re-ruptured before clipping, most in the operating room.

When there are controversies regarding the interpretation of clinical efficacy data, other considerations, including cost:benefit calculations, durability of the procedure, patient selection criteria, and physician preferences, may sway decisions. Public health planners, medical payers, and governmental agencies are most
The best

approach

often involves collaborative

interactions and decisions

made by a

team

RL Sacco is Miller Professor and Chair of Neurology at the Miller School of Medicine, University of Miami, Miami, FL, USA, and an Advisory Board member of Nature Clinical Practice Neurology.

\section{Competing interests}

The author declared no competing interests.

www.nature.com/clinicalpractice doi:10.1038/ncpneuro0828 persuaded by cost analyses. The costs of a new technology may at first be greater than those of the older technology. However, a Practice Point in this issue considers recent evidence that the overall costs at 1 and 2 years are comparable between clipping and coiling. Total cost cannot, therefore, be used as a strong reason to choose one of these treatments over the other. Similar cost but better efficacy, however, will translate into a cost:utility analysis in favor of coiling, because this measure will be driven by the efficacy results.

When comparing two treatment options we must also consider the technical adequacy (durability) of the treatment to obliterate the aneurysm. This issue also contains a Practice Point that discusses predictors of long-term re-rupture after treatment of cerebral aneurysms. The most important re-rupture predictor was the degree of aneurysm occlusion, which was better more often after clipping than coiling. In ISAT, late re-treatment of aneurysms was 6.9 times more likely after coiling, and younger age, larger lumen size, and incomplete occlusion were found to be risk factors for late re-treatment (Camp A et al. [2007] Stroke 38: 1538-1544). Moreover, in ISAT, clipping was found to be superior to coiling among patients younger than 40 years. Regardless of which procedure is used to treat a leaking cerebral aneurysm, the key to long-term success will be the achievement of more-complete occlusion of the aneurysm.

Physician availability and preference may also influence therapeutic decision-making when controversy exists. The technical proficiency of the local practicing clinicians may help the decision of which procedure to choose to treat a ruptured aneurysm. The best approach often involves collaborative interactions and decisions made by a team. Like any emerging treatment, however, technologies, practice and endovascular skills will only continue to improve over time and will probably lead to more coiled than clipped aneurysms in the future. 